\title{
Kinetic Equation for Gluons in the Background Gauge of QCD
}

\author{
Q. Wang, ${ }^{1,2, *}$ K. Redlich, ${ }^{3,4, \dagger}$ H. Stöcker, ${ }^{1,+}$ and W. Greiner ${ }^{1, \S}$ \\ ${ }^{1}$ Institute für Theoretische Physik, J. W. Goethe-Universität, D-60054 Frankfurt, Germany \\ ${ }^{2}$ Physics Department, Shandong University, Jinan, Shandong 250100, People's Republic of China \\ ${ }^{3}$ Theory Division, CERN, CH-1211 Geneva 23, Switzerland \\ ${ }^{4}$ Institute for Theoretical Physics, University of Wroclaw, PL-50204 Wroclaw, Poland
}

(Received 13 November 2001; published 19 March 2002)

\begin{abstract}
We derive the quantum kinetic equation for a pure gluon plasma, applying the background field and closed-time-path method. The derivation is more general and transparent than earlier works. A term in the equation is found which, as in the classical case, corresponds to the color charge precession for partons moving in the gauge field.
\end{abstract}

DOI: $10.1103 /$ PhysRevLett.88.132303

PACS numbers: 12.38.Mh, 11.15.Kc, 24.85.+p, 25.75.-q

Heavy ion collisions at ultrarelativistic energies are widely expected to be a laboratory to study the formation and properties of highly excited QCD matter in the deconfined quark-gluon plasma (QGP) phase [1]. The QGP is considered as a partonic system being at (or close to) local thermal equilibrium. Thus, to study the conditions for the possible formation of QGP in heavy ion collisions one needs to address the question of thermalization of the initially produced partonic medium [2]. In the theoretical aspect this requires the formulation of the kinetic equations [3] involving color degrees of freedom and the nonAbelian structure of QCD dynamics. Various models for the initial conditions in ultrarelativistic heavy ion collisions suggest that at the early stage the medium is dominated by gluon degrees of freedom [4]. The kinetic equation for a pure gluon plasma is thus of special interest.

The usual treatment of the gluon transport equation is based on the decomposition of the gluon field into a mean field and a quantum fluctuation. Under this approximation the gluon transport equation then describes the kinetics of the quanta in the classical mean field [5-7]. This picture is somewhat similar to the one used in studying the energy loss of a fast parton moving in the soft mean field $[8,9]$. To include the classical chromofield into QCD in a proper way, one uses the background field method of QCD (BGQCD) introduced by DeWitt and 't Hooft [10-12]. The advantage of BG-QCD is that it is formulated in an explicit gauge invariant manner.

One of the first attempts to derive the gluon transport equation in BG-QCD was presented in Ref. [13]. But the obtained equation is not transparent. The most recent work has been done by Blaizot and Iancu $[14,15]$ in the closedtime-path (CTP) formalism. There, however, the authors focus on formulating the transport equation in the vicinity of equilibrium.

In this paper, we use the CTP and BG-QCD methods to derive the kinetic equation for the gluon plasma. Our derivation is going beyond previous results as it is quite general and transparent. One of the most important features of our work is that a term in the obtained equation is shown to correspond to the color charge precession term in the classical equation [5,16-19].

In the following we use $g_{\mu \nu}=\operatorname{diag}(1,-1,-1,-1)$ as the metric tensor, and for elegance of the formula we always write Lorentz indices as subscripts and color ones as superscripts for the relevant quantities. For the gauge field and its strength tensor we denote $A_{\mu} \equiv A_{\mu}^{a} T^{a}$ and $F_{\mu \nu}[A] \equiv F_{\mu \nu}^{a}[A] T^{a}$, where $\left(T^{a}\right)^{i j}=i f^{i a j}$ are the generators of the $\mathrm{SU}(3)_{c}$ adjoint representation. The twopoint Green function (GF) or self-energy (SE) are treated as matrices, so their color and/or Lorentz indices are sometimes omitted.

Applying the background field method, we decompose the conventional gluon field into the sum of a classical background part $A$ and a quantum fluctuation $Q$. Including the appropriate gauge fixing and ghost terms for the background gauge $D_{\mu}^{i j}[A] Q_{\mu}^{j}=0$, the BG-QCD Lagrangian reads [12]

$$
\begin{aligned}
\mathcal{L}= & -\frac{1}{4} F_{\mu \nu}^{i}[A+Q] F_{\mu \nu}^{i}[A+Q]-\frac{1}{2 \alpha}\left(D_{\mu}^{i j}[A] Q_{\mu}^{j}\right)^{2} \\
& +\bar{C}^{i} D_{\mu}^{i j}[A] D_{\mu}^{j k}[A+Q] C^{k},
\end{aligned}
$$

where $D_{\mu}^{i j}[A(x)]=\left[\partial_{x \mu}-i g A_{\mu}(x)\right]^{i j}$ is the covariant derivative, $C^{i} / \bar{C}^{i}$ are the ghost/antighost field and $\alpha$ is the gauge fixing parameter.

The above Lagrangian is invariant under the local gauge transformation of type I (type II transformation is irrelevant to the current problem) where the background field transforms as conventional gauge fields, $A_{\mu}^{\prime}=$ $U A_{\mu} U^{-1}+i g^{-1} U \partial_{\mu} U^{-1}$, while the gluon and ghost fields transform like a matter field, $Q_{\mu}^{\prime}=U Q_{\mu} U^{-1}$ [12]. Here $U(x)=\exp \left[\operatorname{ig} \omega^{a}(x) T^{a}\right]$ is the transformation matrix.

The nonequilibrium dynamics is usually described in the CTP formalism [20]. Here the action can be written as $S_{\text {CTP }}=S_{+}-S_{-}+K\left(A_{ \pm}, Q_{ \pm}\right)$where all fields in $S_{ \pm}$ are defined on the positive/negative time branches and $K\left(A_{ \pm}, Q_{ \pm}\right)$is the kernel incorporating initial state correlations. The role of the nonlocal kernel $K\left(A_{ \pm}, Q_{ \pm}\right)$in $S_{\mathrm{CTP}}$ 
is discussed in Ref. [21] and its connection with the pinch singularity [22] will be discussed elsewhere. The GF for the gluon has four components, $\left[G^{++}, G^{+-}, G^{-+}\right.$, $\left.G^{--}\right] \equiv\left[G^{F}, G^{<}, G^{>}, G^{\bar{F}}\right]$, marked by the positive or the negative time branch, respectively. One can also express the GF in the physical representation, where the GF is expressed by the symmetric (C), retarded (R), and advanced (A) components. The two representations are related by a unitary transformation. The same expressions for the SE are also valid.

In the CTP formalism, choosing the physical representation for the GF and SE, the Dyson-Schwinger equation (DSE) for $G^{C}$ reads

$$
\begin{aligned}
\mathcal{D}\left(x_{1}\right) G^{C}\left(x_{1}, x_{2}\right)=-\int d^{4} x^{\prime}\left[\Pi^{C}\left(x_{1}, x^{\prime}\right) G^{A}\left(x^{\prime}, x_{2}\right)\right. \\
\left.+\Pi^{R}\left(x_{1}, x^{\prime}\right) G^{C}\left(x^{\prime}, x_{2}\right)\right],
\end{aligned}
$$

$$
\begin{aligned}
G^{C}\left(x_{1}, x_{2}\right) \mathcal{D}^{\dagger}\left(x_{2}\right)=-\int d^{4} x^{\prime}[ & G^{R}\left(x_{1}, x^{\prime}\right) \Pi^{C}\left(x^{\prime}, x_{2}\right) \\
+ & \left.G^{C}\left(x_{1}, x^{\prime}\right) \Pi^{A}\left(x^{\prime}, x_{2}\right)\right]
\end{aligned}
$$

where the differential operators $\mathcal{D}$ and $\mathcal{D}^{\dagger}$ in the Feynman gauge [23] $(\alpha=1)$ are expressed by

$$
\begin{array}{r}
\mathcal{D}_{\rho \sigma}^{h i}=g_{\rho \sigma} D_{\mu}^{h a}[A] D_{\mu}^{a i}[A]+2 g f^{h a i} F_{\rho \sigma}^{a}[A], \\
\mathcal{D}_{\rho \sigma}^{\dagger ; h i}=g_{\rho \sigma} D_{\mu}^{\dagger ; h a}[A] D_{\mu}^{\dagger ; a i}[A]+2 g f^{h a i} F_{\rho \sigma}^{a}[A],
\end{array}
$$

where $D_{\mu}^{\dagger ; i j}[A(x)] \equiv\left[\overleftarrow{\partial}_{x \mu}+i g A_{\mu}(x)\right]^{i j}$ is the conjugate covariant derivative where the differential operator acts on the function in its left.

In the evolution of the gluonic system one distinguishes different scales, which characterize quantum and soft col- lective motion. We introduce a mass parameter, $\mu$, as the separation point of the quantum and the kinetic scales. In the weak coupling limit $g \ll 1$, the scale of collectivity $\sim 1 /(g \mu)$ is much larger than the typical extension of hard quantum fluctuations $\sim 1 / \mu$. The effect of the classical field $A$ on the hard quanta involves the coupling $g A$ to the hard propagator and is of the size of the soft wavelength $\sim 1 /(g \mu)$. The above separation of scales is the basis for the gradient expansion where one expresses all two-point GFs in terms of the relative $y$ and the central $X$ coordinate. Here are some typical scales: $y=x_{1}-$ $x_{2} \sim 1 / \mu, X=\left(x_{1}+x_{2}\right) / 2 \sim 1 / g \mu, A(X) \sim \mu$, and $F[A(X)] \sim g \mu^{2}$.

In order to obtain the gauge-covariant kinetic equation one uses the gauge-covariant Wigner function $\tilde{G}(X, y)$ $\left(\tilde{G}^{C}, \tilde{G}^{>}\right.$, or $\left.\tilde{G}^{<}\right)$defined by

$$
G\left(x_{1}, x_{2}\right)=V\left(x_{1}, X\right) \tilde{G}(X, y) V\left(X, x_{2}\right),
$$

where $V\left(z_{1}, z_{2}\right)=\mathrm{T}_{\mathrm{P}} \exp \left(i g \int_{\mathrm{P}_{;} z_{2}}^{z_{1}} d z_{\mu} A_{\mu}\right)$ denotes a Wilson link with respect to the classical background field. One can also define the Wilson link as a functional of $A+Q$, but it is a much more complicated case and beyond the scope of this work.

The covariant Wigner function $\tilde{G}(X, y)$ transforms as $U(X) \tilde{G}(X, y) U^{-1}(X)$ where only the transformation at a single point $X$ is relevant. For $G\left(x_{1}, x_{2}\right)$, however, the gauge transformation involves two points and therefore is not gauge-covariant.

The DSE given by Eqs. (2) and (3) can be expressed in terms of the covariant Wigner functions. To evaluate $\mathcal{D}\left(x_{1}\right) G\left(x_{1}, x_{2}\right)$ one needs to know the variation of a Wilson link caused by that of its ending points. Following Eq. (3.15) for $\delta V\left(z_{1}, z_{2}\right)$ in Ref. [17] (note the opposite sign convention for $g$ ), one finds

$$
\begin{gathered}
D_{x_{1} \nu}\left(V_{1} \tilde{G} V_{2}\right) \approx V_{1}\left[\left(\partial_{y \nu} \tilde{G}\right)+\frac{1}{2}\left(D_{X \nu} \tilde{G}\right)+i g \frac{1}{2} \tilde{G} A_{\nu}-i g \frac{3}{8} y_{\lambda} F_{\lambda \nu} \tilde{G}-i g \frac{1}{8} \tilde{G} y_{\lambda} F_{\lambda \nu}\right] V_{2}, \\
D_{x_{1}}^{2}\left(V_{1} \tilde{G} V_{2}\right) \approx V_{1}\left[\frac{1}{4}\left(D_{X}^{2} \tilde{G}\right)+\left(\partial_{y} \cdot D_{X} \tilde{G}\right)+\left(\partial_{y}^{2} \tilde{G}\right)-i g \frac{3}{4} y_{\lambda} F_{\lambda \nu}\left(\partial_{y \nu} \tilde{G}\right)-i g \frac{1}{4}\left(\partial_{y \nu} \tilde{G}\right) y_{\lambda} F_{\lambda \nu}+i g\left(\partial_{y \nu} \tilde{G}\right) A_{\nu}\right. \\
\left.-i g \frac{3}{8} y_{\lambda} F_{\lambda \nu}\left(D_{X \nu} \tilde{G}\right)+i g \frac{1}{2}\left(D_{X \nu} \tilde{G}\right) A_{\nu}+i g \frac{1}{4} \tilde{G}\left(\partial_{X \nu} A_{\nu}\right)-i g \frac{1}{8}\left(D_{X \nu} \tilde{G}\right) y_{\lambda} F_{\lambda \nu}-\frac{1}{4} g^{2} \tilde{G} A^{2}\right] V_{2},
\end{gathered}
$$

where we have used $V_{1} \equiv V\left(x_{1}, X\right), V_{2} \equiv V\left(X, x_{2}\right), \tilde{G} \equiv$ $\tilde{G}(X, y), D_{X \nu} \equiv D_{\nu}[A(X)], D_{t}^{2} \equiv D_{t \nu} D_{t \nu}, A_{\mu} \equiv A_{\mu}(X)$, $A^{2} \equiv A_{\mu}(X) A_{\mu}(X)$, and $F_{\mu \nu} \equiv F_{\mu \nu}(X)$. The conjugate expressions $\left(V_{1} \tilde{G} V_{2}\right) D_{x_{2} \nu}^{\dagger}$ and $\left(V_{1} \tilde{G} V_{2}\right) D_{x_{2}}^{\dagger 2}$ can be obtained from Eqs. (7) and (8) by taking their Hermitian conjugates and then interchanging $x_{1}$ and $x_{2}$. Using these results together with Eq. (6) we derive the following gauge conditions for $\tilde{G}$ [24]:

$$
\partial_{X \mu} \tilde{G}_{\mu \nu}+i g\left[\tilde{G}_{\mu \nu}, A_{\mu}\right]=0, \quad \partial_{y \mu} \tilde{G}_{\mu \nu}=0,
$$

which are valid up to $O(g \mu)$. The second equation is a transversality condition for $\tilde{G}_{\mu \nu}$.

Taking the difference between Eq. (2) and Eq. (3) and using Eq. (8) and its conjugate expression, we derive the following kinetic equation for the gluon plasma:

$$
\begin{aligned}
& q \cdot \partial_{X} \tilde{G}_{\alpha \gamma}^{C}+i g\left(\tilde{G}_{\alpha \gamma}^{C} q \cdot A-q \cdot A \tilde{G}_{\alpha \gamma}^{C}\right)+ \\
& \frac{1}{2} g q_{\nu} F_{\nu \lambda}\left(\partial_{q \lambda} \tilde{G}_{\alpha \gamma}^{C}\right)+\frac{1}{2} g\left(\partial_{q \lambda} \tilde{G}_{\alpha \gamma}^{C}\right) q_{\nu} F_{\nu \lambda}+ \\
& \quad g\left(F_{\alpha \beta} \tilde{G}_{\beta \gamma}^{C}-\tilde{G}_{\alpha \beta}^{C} F_{\beta \gamma}\right)=0,
\end{aligned}
$$

where $\tilde{G}_{\alpha \gamma}^{C} \equiv \tilde{G}_{\alpha \gamma}^{C}(X, q)$.

The above equation is located at the collective coordinate $X$ and is gauge-covariant under the local gauge transformation $U(X)$; i.e., it transforms as $U(\cdots) U^{-1}$. Indeed, noting that both $F_{\mu \nu}$ and $\tilde{G}_{\alpha \gamma}^{C}$ are gauge-covariant and $\partial_{q \mu}$ does not affect $U$, it is obvious that the last three terms are gauge-covariant. To verify that the first two terms also preserve gauge covariance, we explicitly write down their 
transformation

$$
\begin{gathered}
q \cdot \partial_{X} \tilde{G}_{\alpha \gamma}^{C} \rightarrow U\left(q \cdot \partial_{X} \tilde{G}_{\alpha \gamma}^{C}\right) U^{-1}+\left(q \cdot \partial_{X} U\right) \tilde{G}_{\alpha \gamma}^{C} U^{-1}+U \tilde{G}_{\alpha \gamma}^{C} q \cdot \partial_{X} U^{-1}, \\
i g \tilde{G}_{\alpha \gamma}^{C} q \cdot A \rightarrow i g U \tilde{G}_{\alpha \gamma}^{C} q \cdot A U^{-1}-U \tilde{G}_{\alpha \gamma}^{C} q \cdot \partial_{X} U^{-1} \\
-i g q \cdot A \tilde{G}_{\alpha \gamma}^{C} \rightarrow-i g U q \cdot A \tilde{G}_{\alpha \gamma}^{C} U^{-1}-\left(q \cdot \partial_{X} U\right) \tilde{G}_{\alpha \gamma}^{C} U^{-1}
\end{gathered}
$$

With the above gauge transformations one can clearly see that the sum of the first two terms in Eq. (10) indeed transforms as $U(\cdots) U^{-1}$ and therefore preserves the gauge covariance.

The quantum kinetic equation (10) is derived in a quite general and consistent manner in BG-QCD and CTP formalism. No further approximations or requirements going beyond the gradient expansion were used. We notice that a result similar to Eq. (10) was also obtained in Ref. [25]. There, however, the kinetic equation was derived by making the gradient expansion of the equation of motion for the Wigner function (not in CTP formalism). In addition, the derivation was made in the fundamental (not the adjoint) color space in QCD (not BG-QCD). Finally, the assumption was made in Ref. [25] that the Wigner function is proportional to the quadratic product of the generators of the fundamental representation. The approach presented here is quite general and does not require any specific assumptions on the structure of the Wigner function.

In the following we will compare Eq. (10) with the classical equation. Especially, we will show the physical connection of the second term of Eq. (10) to the color precession. The classical kinetic equation for the color singlet distribution function $f(x, p, Q)$ has the following form $[5,16-19]$ :

$$
\begin{array}{r}
p_{\mu}\left[\partial_{\mu}-g Q^{a} F_{\mu \nu}^{a} \partial_{p \nu}-g f^{a b c} A_{\mu}^{b}(x) Q^{c} \partial_{Q^{a}}\right] \times \\
f(x, p, Q)=0,
\end{array}
$$

where $Q^{a}$ is the classical color charge and $a=$ $1, \ldots, N_{c}^{2}-1$. The last term of Eq. (11) describes the rotation or precession of the color charge $[5,16,17]$.

Comparing Eq. (11) with the quantum expression Eq. (10), it is clear that the color singlet distribution function $f$ is replaced by the gauge-covariant Wigner function $\tilde{G}^{C}$ which is a color matrix in the adjoint representation. One can also recognize that the first, third, and fourth terms of Eq. (10) are the quantum generalization of the first two terms in Eq. (11). The last term in Eq. (10) appears from the covariant operators [26] and hence is not present in the classical equation.

Particularly interesting is the appearance of the second term in Eq. (10). We have seen that its presence is crucial to assure the gauge covariance of the Vlasov equation. This term has an interesting physical meaning. It is the quantum analog to the color charge precession in the classical kinetic equation. To see this more clearly, one can expand $\tilde{G}_{\alpha \beta}^{C}(X, q)$ with respect to the expansion parameter $g T^{a} A_{\mu}^{a}(X)$ from the Wilson link in Eq. (6). This expansion can also be understood as the result of the $A Q Q$, $A Q Q Q$, and $A A Q Q$ vertices. Then we have

$$
\begin{aligned}
\tilde{G}_{\alpha \beta}^{C}(X, q)= & N_{0 \alpha \beta}(X, q)+g T^{a} A_{\mu}^{a}(X) N_{1 \alpha \beta ; \mu}(X, q) \\
& +g^{2} T^{a} T^{b} A_{\mu}^{a}(X) A_{\nu}^{b}(X) N_{2 \alpha \beta ; \mu \nu}(X, q) \\
& +\ldots,
\end{aligned}
$$

where $T^{a}$ are quantum analogs to the classical color charges $Q^{a} ; N_{i \alpha \beta}(X, q)$ with $i=0,1,2, \ldots$ are color singlet functions. Each term of the expansion corresponds to an order of the color inhomogeneity in the gluonic medium due to its interaction with the background field. If the background field is referred to the soft mean field, its magnitude should vanish when the system approaches equilibrium. Then only the first singlet term survives in Eq. (12), which means the color homogeneity of the gluonic medium. This is somewhat similar to the multipole expansion for an electromagnetic source where the moments of dipole, quadrupole, etc., describe increasing orders of spatial inhomogeneity for the electromagnetic charges. In weak coupling, as the lowest order approximation, we keep only the first two terms in Eq. (12). Then the second term of Eq. (10) becomes

$$
i g\left(\tilde{G}_{\alpha \gamma}^{C} q \cdot A-q \cdot A \tilde{G}_{\alpha \gamma}^{C}\right) \simeq-g f^{a b c} q_{\mu} A_{\mu}^{b} T^{c} \partial_{T^{a}} \tilde{G}_{\alpha \gamma}^{C},
$$

which reproduces the classical color precession term in Eq. (11).

Since we know that $D_{X} \sim g A(X) \sim g \mu$, the first two terms of Eq. (10), i.e., $q \cdot \partial_{X} \tilde{G}_{\alpha \gamma}^{C}$ and the color precession term, are at leading order $O\left(g \mu^{2}\right)$, while other terms are at subleading order $O\left(g^{2} \mu^{2}\right)$. In the vicinity of equilibrium the natural scale in the system is the temperature $T$. The mean distance between particles is of the order of $\sim 1 / T$, while $1 /(g T)$ characterizes the scale of collective excitations $[14,15]$. For small coupling constant $g$ these two scales are well separated. The covariant Wigner functions can be expanded around their equilibrium values, $\tilde{G}=$ $\tilde{G}^{(0)}+\delta \tilde{G}$, where the equilibrium function $\tilde{G}^{(0)}$ is a color singlet and the fluctuation $\delta \tilde{G} \sim g^{2} \tilde{G}$. Typical scales are $q \sim T, D_{X} \sim g^{2} T, g F \sim\left(D_{X}\right)^{2} \sim g^{4} T^{2}$. Thus, at leading order, only the first term of Eq. (10) survives, and the precession term vanishes due to the color-singlet nature of $\tilde{G}^{(0)}$. The linearized version of Eq. (10) with respect to $\delta \tilde{G}$ corresponds to the equation formulated in the background Coulomb gauge in Ref. [14].

The quantum fluctuations near equilibrium were also considered in Ref. [27] in the context of the classical collisionless transport equation. It is quite natural to carry out the same study from our quantum approach. First, BGQCD deals with the classical field and the quantum fluctuation in a systematic way. The quantum field plays the similar role to the field fluctuation in Ref. [27]. Second, in 
the quantum approach, corresponding to the phase-space distribution, we deal with the GFs which can be expanded around their equilibrium values following Eq. (12). One also needs to complete the equations by including the field equation $D_{\mu} F_{\mu \nu}=\left\langle J_{\nu}\right\rangle$. Here the averaged induced current $\left\langle J_{\nu}\right\rangle$ is related to the two- and three-point GFs [15] which finally depend on $\tilde{G}$. Thus, it can be expanded according to Eq. (12) as well.

The analogy and differences of the quantum and the classical Vlasov equation can also be shown by comparing the equations for the color moments. Corresponding to Eq. (10) one gets

$$
\begin{gathered}
q \cdot \partial_{X} h_{\alpha \gamma}+g q_{\nu} F_{\nu \lambda}^{a} \partial_{q \lambda} h_{\alpha \gamma}^{a}+ \\
g\left(F_{\alpha \beta}^{a} h_{\beta \gamma}^{a}-h_{\alpha \beta}^{a} F_{\beta \gamma}^{a}\right)=0, \\
q \cdot \partial_{X} h_{\alpha \gamma}^{a}+g f^{a b c} q \cdot A^{b} h_{\alpha \gamma}^{c}+ \\
g q_{\nu} F_{\nu \lambda}^{b} \partial_{q \lambda} \frac{1}{2}\left[h_{\alpha \gamma}^{a b}+h_{\alpha \gamma}^{b a}\right]+ \\
g\left(F_{\alpha \beta}^{b} h_{\beta \gamma}^{a b}-h_{\alpha \beta}^{b a} F_{\beta \gamma}^{b}\right)=0,
\end{gathered}
$$

where we defined $h_{\alpha \gamma}=\operatorname{Tr}\left(\tilde{G}_{\alpha \gamma}^{C}\right), h_{\alpha \gamma}^{a}=\operatorname{Tr}\left(T^{a} \tilde{G}_{\alpha \gamma}^{C}\right)$, and $h_{\alpha \gamma}^{a b}=\operatorname{Tr}\left(T^{a} T^{b} \tilde{G}_{\alpha \gamma}^{C}\right)$. The classical equations for color moments of $f(x, p, Q)$ can be found in Ref. [5]. Comparing Eqs. (14) and (15) with the classical expressions in Ref. [5], one sees that apart from the term $\left(F_{\alpha \beta} h_{\beta \gamma}-h_{\alpha \beta} F_{\beta \gamma}\right)$, which comes from the covariant operators, the quantum and classical equations have similar structure. The identification of the color precession term in Eq. (10) is then straightforward.

In summary, by applying the CTP and BG-QCD formalism we have derived the kinetic equation for the gluon. The derivation is more general and transparent than earlier works. The kinetic equation is with respect to gauge-covariant Wigner function, which is a matrix in adjoint color space. A notable feature of our work is that a term is found which, as in the classical case, corresponds to the color precession of the parton. This is the nonAbelian analog to the Larmor precession for particles with the magnetic moment moving in a magnetic field. We see that this term is necessary to the gauge covariance of the kinetic equation.

One of us (Q. W.) acknowledges financial support of the Alexander von Humboldt Foundation $(\mathrm{AvH})$ and appreciates help from D. Rischke. This work is partially funded by DFG, BMBF, and GSI. K. R. acknowledges partial support of the Polish Committee for Scientific Research (KBN-2P03B 03018). Stimulating comments and discussions with R. Baier, J.-P. Blaizot, E. Iancu, and S. Leupold are acknowledged. Our special thanks go to X.-N. Wang for his help and interest in this work.

*Electronic address: qwang@th.physik.uni-frankfurt.de

${ }^{\dagger}$ Electronic address: Krzysztof.Redlich@cern.ch

†Electronic address: stoecker@th.physik.uni-frankfurt.de

${ }^{\S}$ Electronic address: greiner@th.physik.uni-frankfurt.de

[1] For a recent review, see, e.g., S. A. Bass, M. Gyulassy,
H. Stocker, and W. Greiner, J. Phys. G 25, R1 (1999); H. Satz, Rep. Prog. Phys. 63, 1511 (2000).

[2] R. Baier, A. H. Mueller, D. Schiff, and D. T. Son, Phys. Lett. B 502, 51 (2001).

[3] For a recent review, see, e.g., J.-P. Blaizot and E. Iancu, hep-ph/0101103 [Phys. Rep. (to be published)].

[4] L. McLerran and R. Venugopalan, Phys. Rev. D 49, 2233 (1994); 49, 3352 (1994); 50, 2225 (1994); K. J. Eskola, K. Kajantie, P. V. Ruuskanen, and K. Tuominen, Nucl. Phys. B570, 379 (2000).

[5] U. Heinz, Ann. Phys. (N.Y.) 161, 48 (1985); 168, 148 (1986); H.-Th. Elze and U. Heinz, Phys. Rep. 183, 81 (1989).

[6] H.-Th. Elze, Z. Phys. C 38, 211 (1988).

[7] K. Geiger, Phys. Rev. D 56, 2665 (1997).

[8] M. Gyulassy and X.-N. Wang, Nucl. Phys. B420, 583 (1994); M. Gyulassy, X.-N. Wang, and M. Plümer, Phys. Rev. D 51, 3436 (1995); X.-F. Guo and X.-N. Wang, Phys. Rev. Lett. 85, 3591 (2000).

[9] R. Baier et al., Nucl. Phys. B483, 291 (1997); B484, 265 (1997); R. Baier et al., Nucl. Phys. B531, 403 (1997).

[10] B.S. DeWitt, Phys. Rev. 162, 1195 (1967); 162, 1239 (1967).

[11] G. 't Hooft, in Acta Universitatis Wratislaviensis No. 368, Karpacz, 1975 (University of Wroclaw Press, Wroclaw, 1976), Vol. 1, pp. 345-369

[12] L. F. Abbott, Nucl. Phys. B185, 189 (1981); R. B. Sohn, Nucl. Phys. B273, 468 (1986); H. Kluberg-Stern and J. B. Zuber, Phys. Rev. D 12, 482 (1975).

[13] H.-Th. Elze, Z. Phys. C 47, 647 (1990).

[14] J.-P. Blaizot and E. Iancu, Nucl. Phys. B557, 183 (1999).

[15] J.-P. Blaizot and E. Iancu, Phys. Rev. Lett. 70, 3376 (1993); Nucl. Phys. B417, 608 (1994); B570, 326 (2000).

[16] S. K. Wong, Nuovo Cimento A 65, 689 (1970).

[17] H.-Th. Elze, M. Gyulassy, and D. Vasak, Nucl. Phys. B276, 706 (1986).

[18] J. Jalilian-Marian, S. Jeon, R. Venugopalan, and J. Wirstam, Phys. Rev. D 62, 045020 (2000).

[19] P. R. Kelly, Q. Liu, C. Lucchesi, and C. Manuel, Phys. Rev. Lett. 72, 3461 (1994).

[20] J. Schwinger, J. Math. Phys. (N.Y.) 2, 407 (1961); L. V. Keldysh, Sov. Phys. JETP 20, 1018 (1965); G. Z. Zhou, Z. B. Su, B. L. Hao, and L. Yu, Phys. Rep. 118, 1 (1985); E. Calzetta and B. L. Hu, Phys. Rev. D 37, 2878 (1988).

[21] Q. Wang, K. Redlich, H. Stöcker, and W. Greiner (to be published).

[22] C. Greiner and S. Leupold, Eur. Phys. J. C 8, 517 (1999).

[23] Because of gauge conditions, $D_{\mu}^{i j}\left[A\left(x_{1}\right)\right] G_{\mu \nu}^{j k}\left(x_{1}, x_{2}\right)=0$ and $G_{\mu \nu}^{i j}\left(x_{1}, x_{2}\right) D_{\nu}^{\dagger ; j k}\left[A\left(x_{2}\right)\right]=0$, Eqs. (2) and (3) are independent of the gauge parameter $\alpha$.

[24] To derive these results one needs the following gauge conditions for $G\left(G^{>}, G^{<}\right.$, or $\left.G^{C}\right)$ with respect to $x_{1}$ and $x_{2}$ : $D_{\mu}\left[A\left(x_{1}\right)\right] G_{\mu \nu}\left(x_{1}, x_{2}\right)=0$ and $G_{\mu \nu}\left(x_{1}, x_{2}\right) D_{\nu}^{\dagger}\left[A\left(x_{2}\right)\right]=0$. They are consequences of the background gauge condition $D_{\mu}[A(x)] Q_{\mu}(x)=0$.

[25] S. Mrowczynski, Phys. Rev. D 39, 1940 (1989).

[26] This term can be written in a different form by using generators of the Lorentz transformation in vector representation. A similar term can be found for the quark, but expressed through generators in spinor representation.

[27] D. F. Litim and C. Manuel, Phys. Rev. Lett. 82, 4981 (1999). 